\title{
MAPS PRESERVING GENERAL MEANS OF POSITIVE OPERATORS*
}

\author{
LAJOS MOLNÁR ${ }^{\dagger}$
}

\begin{abstract}
Under some mild conditions, the general form of bijective transformations of the set of all positive linear operators on a Hilbert space which preserve a symmetric mean in the sense of Kubo-Ando theory is described.
\end{abstract}

Key words. Positive operators, Mean, Automorphisms, Preservers.

AMS subject classifications. 47B49, 47A64.

1. Introduction. Let $H$ be a complex Hilbert space and denote by $B(H)$ the algebra of all bounded linear operators on $H$. An operator $A \in B(H)$ is said to be positive if $\langle A x, x\rangle \geq 0$ holds for every vector $x \in H$. The cone of all positive operators on $H$ is denoted by $B(H)^{+}$.

In our recent papers [5,6], we have described the structure of all bijective maps $\phi$ on $B(H)^{+}$(assuming $\operatorname{dim} H \geq 2$ ) which preserve either the geometric mean \# or the harmonic mean! of operators. For example, in the case of the geometric mean, this means that $\phi(A \# B)=\phi(A) \# \phi(B)$ holds for any $A, B \in B(H)^{+}$. We have proved that all those transformations are of the form $\phi(A)=T A T^{*}, A \in B(H)^{+}$with an invertible bounded linear or conjugate-linear operator $T: H \rightarrow H$.

However, there is a general abstract theory of operators means due to Kubo and Ando [2] which has important applications in several areas, from operator theory to quantum information theory (cf. the theory of monotone metrics due to Petz $[8]$ ). In this paper, we extend our previous investigations and study the structure of all bijective transformations of $B(H)^{+}$which preserve general operator means. Unfortunately, we do not have a result in the most general sense, i.e., relating to arbitrary means on $B(H)^{+}$and arbitrary bijective transformations on $B(H)^{+}$with the mean-preserving property. In fact, we have to pose a mild condition on the means

*Received by the editors on March 8, 2011. Accepted for publication on August 14, 2011. Handling Editor: Moshe Goldberg.

$\dagger$ Institute of Mathematics, University of Debrecen, H-4010 Debrecen, PO Box 12, Hungary (molnarl@science.unideb.hu, http://www.math.unideb.hu/ molnarl/). The author was supported by the Hungarian Scientific Research Fund (OTKA) K81166 NK81402, and by the TÁMOP 4.2.1./B09/1/KONV-2010-0007 project implemented through the New Hungary Development Plan cofinanced by the European Social Fund and the European Regional Development Fund. 
(symmetry) and some mild regularity assumption (e.g. a sort of continuity) on the transformations. As for the main idea of the proof, it is based on the knowledge of the structure of the order automorphisms of $B(H)^{+}$that was determined in our paper [3] (alternatively, see Section 2.5 in the book [4]). Some recent applications of that result to other problems can be found in the paper [7].

Now, let us summarize the basic notions and results of Kubo-Ando theory that we shall need in our investigations. In what follows, $H$ denotes a complex Hilbert space with $\operatorname{dim} H \geq 2$. A binary operation $\sigma$ on $B(H)^{+}$is said to be a connection if the following requirements are fulfilled (from (i) to (iii) all operators are supposed to belong to $\left.B(H)^{+}\right)$.

(i) If $A \leq C$ and $B \leq D$, then $A \sigma B \leq C \sigma D$.

(ii) $C(A \sigma B) C \leq(C A C) \sigma(C B C)$.

(iii) If $A_{n} \downarrow A$ and $B_{n} \downarrow B$ strongly, then $A_{n} \sigma B_{n} \downarrow A \sigma B$ strongly.

If $I \sigma I=I$ holds too, then the connection $\sigma$ is called a mean. A mean $\sigma$ is called symmetric if $A \sigma B=B \sigma A$ holds for all $A, B \in B(H)^{+}$. Operations like convex combination and order among connections are defined in a natural way.

By the fundamental result Theorem 3.2 in [2], there is an affine order-isomorphism from the class of connections $\sigma$ onto the class of operator monotone functions $f$ : ] $0, \infty[\rightarrow[0, \infty[$ given by the formula $f(t)=\operatorname{I\sigma t} I(t>0)$. For invertible $A, B \in$ $B(H)^{+}$, we have

$$
A \sigma B=A^{1 / 2} f\left(A^{-1 / 2} B A^{-1 / 2}\right) A^{1 / 2}
$$

By property (iii), we obtain that the formula (1.1) extends to any invertible $A \in$ $B(H)^{+}$and arbitrary $B \in B(H)^{+}$.

We shall need the following so-called transfer property of connections. By (ii), we easily deduce that $C(A \sigma B) C=(C A C) \sigma(C B C)$ holds for all invertible $C \in B(H)^{+}$. Now, using polar decomposition, the formula (1.1) and the continuity property (iii), it follows that we have

$$
T(A \sigma B) T^{*}=\left(T A T^{*}\right) \sigma\left(T B T^{*}\right)
$$

for all invertible bounded linear or conjugate-linear operator $T$ on $H$. This trivially implies that $(t A) \sigma(t B)=t(A \sigma B)$ for any $t>0$ and $A, B \in B(H)^{+}$.

We shall also use Theorem 3.3 in [2] which says that for every mean $\sigma$, we have $A \sigma A=A, A \in B(H)^{+}$.

Relating to operator monotone functions, it is well-known that they have a certain 
integral representation. Namely, by Lemma 3.1 in [2], the formula

$$
f(s)=\int_{[0, \infty]} \frac{s(1+t)}{s+t} d m(t), \quad s>0
$$

provides an affine isomorphism from the class of all positive Radon measures $m$ on $[0, \infty]$ onto the set of all operator monotone functions $f:] 0, \infty[\rightarrow[0, \infty[$. Remark that in the above representation, we have $f(0) \doteq \lim _{s \rightarrow 0} f(s)=m(\{0\})$ and $\lim _{s \rightarrow \infty} f(s) / s=m(\{\infty\})$.

Using the formula (1.2), we then obtain an integral representation of any connection $\sigma$. In fact, Theorem 3.4 in [2] tells us that for each connection $\sigma$ on $B(H)^{+}$, there exists a unique positive Radon measure on $[0, \infty]$ such that with $a=m(\{0\})$ and $b=m(\{\infty\})$ we have

$$
A \sigma B=a A+b B+\int_{] 0, \infty[} \frac{1+t}{t}\{(t A: B)\} d m(t)
$$

for all $A, B \in B(H)^{+}$(here and below : stands for the parallel sum of positive operators which is the half of the harmonic mean). Moreover, the correspondence $\sigma \mapsto m$ is an affine isomorphism from the class of all connections onto the class of all positive Radon measures on $[0, \infty]$.

Below whenever we write $\sigma, f, m$ we always mean that $\sigma$ is a connection, $f$ is its representing operator monotone function and $m$ is its representing positive Radon measure.

2. Results and proofs. In what follows, $\sigma$ denotes a symmetric mean with the property that $I \sigma 0=0 \sigma I=0$. This means that in (1.3) we have $a=m(\{0\})=f(0)=$ 0 . Taking into account that symmetry of $\sigma$ means that $f(s)=s f(1 / s)$ holds for all $s>0$ (see Corollary 4.2. in [2]), we obtain that $b=m(\{\infty\})=\lim _{s \rightarrow \infty} f(s) / s=$ $\lim _{s \rightarrow 0} f(s)=f(0)=0$. Consequently, the integral representation of $\sigma$ is

$$
A \sigma B=\int_{] 0, \infty[} \frac{1+t}{t}\{(t A: B)\} d m(t), \quad A, B \in B(H)^{+} .
$$

There is still another fact that we shall need: for any operator $A \in B(H)^{+}$we have $I \sigma A=f(A)($ see $(3.7)$ in $[2])$.

THEOREM 2.1. Let $\phi: B(H)^{+} \rightarrow B(H)^{+}$be a bijective map that satisfies

$$
\phi(A \sigma B)=\phi(A) \sigma \phi(B)
$$

for all $A, B \in B(H)^{+}$. If there exists an invertible operator $A \in B(H)^{+}$such that either $\phi$ is continuous on the set of scalar multiples $t A, t \geq 0$ of $A$, or it maps any 
scalar multiple $t A, t \geq 0$ of $A$ to a scalar multiple of $\phi(A)$, then it follows that $\phi$ is of the form

$$
\phi(A)=T A T^{*}, \quad A \in B(H)^{+}
$$

with some invertible bounded linear or conjugate-linear operator $T: H \rightarrow H$.

Observe that any transformation $\phi$ of the form $\phi(A)=T A T^{*}, A \in B(H)^{+}$with an invertible bounded linear or conjugate-linear operator $T: H \rightarrow H$ satisfies (2.2) and also has both of the regularity properties appearing in the theorem above as assumptions.

For the proof of the theorem, we need some auxiliary results.

Lemma 2.2. For any $A \in B(H)^{+}$, we have that $A$ is a projection if and only if $I \sigma A=A$.

Proof. First we point out that Lemma 5.1 in [2] tells us that for $f$ we have $s<f(s)<1$ for $0<s<1$ and $1<f(s)<s$ for $1<s<\infty$. Now, $f(A)=A$ holds if and only if $f(s)=s$ holds on the spectrum of $A$. This is equivalent to the spectrum of $A$ is in $\{0,1\}$, which means exactly that $A$ is a projection.

Theorem 3.7 in [2] tells us that for any projections $P, Q$ on $H$ we have $P \sigma Q=$ $P \wedge Q$. It follows that $P \leq Q$ if and only if $P \sigma Q=P$.

LEMMA 2.3. We have that $f$ is injective.

Proof. In fact, assuming on the contrary that $f$ is non-injective, it follows that $f$ is constant on some closed interval either before or after the point 1 . In the latter case, there are two more possibilities: the interval can be of finite or infinite length. Using Lemma 5.1 in [2], the concavity of $f(s)$ and the convexity of $s f(s)$ (Lemma 5.2 in [2]) elementary considerations show that we would arrive at contradictions in all cases. This gives us the injectivity of $f$.

Lemma 2.4. Suppose that $f$ is unbounded. Then $A \in B(H)^{+}$is invertible if and only if the equation $A \sigma X=Y$ has a solution $X \in B(H)^{+}$for any given $Y \in B(H)^{+}$.

Proof. Since $f$ is injective and unbounded, we have $f^{-1}:[0, \infty[\rightarrow[0, \infty[$. Suppose that $A$ is invertible. We have learnt from (1.1) that

$$
A \sigma X=A^{1 / 2} f\left(A^{-1 / 2} X A^{-1 / 2}\right) A^{1 / 2}
$$

holds for any $X \in B(H)^{+}$. It requires only easy computation to see that for a given $Y$, defining $X=A^{1 / 2} f^{-1}\left(A^{-1 / 2} Y A^{-1 / 2}\right) A^{1 / 2}$, gives a solution of the equation $A \sigma X=Y$. Assume now that $A$ is not invertible. In that case, for any invertible $X \in B(H)^{+}$, we have

$$
A \sigma X=X \sigma A=X^{1 / 2} f\left(X^{-1 / 2} A X^{-1 / 2}\right) X^{1 / 2} .
$$


Since $f(0)=0$, by the spectral mapping theorem we deduce that the right-hand side of this equality and hence $A \sigma X$, too, are non-invertible. If $X \in B(H)^{+}$is arbitrary, it follows from $A \sigma X \leq A \sigma(X+I)$ that $A \sigma X$ is also not invertible.

Lemma 2.5. Suppose that $f$ is bounded. The operator $A \in B(H)^{+}$is invertible if and only if the set of all operators of the form

$$
\left(\ldots\left(\left(A \sigma T_{1}\right) \sigma T_{2}\right) \ldots\right) \sigma T_{n}, \quad n \in \mathbb{N}, T_{1}, \ldots, T_{n} \in B(H)^{+}
$$

coincides with $B(H)^{+}$.

Proof. To prove the necessity, assume that $A$ is invertible. By the transfer property it is easy to see that there is no serious loss of generality in assuming that $A=I$. Let $\lim _{s \rightarrow \infty} f(s)=r$. Clearly, we have $1<r<\infty$. Pick an $s$ with $1<s$ and let $Y \in B(H)^{+}$. Pick $n \in \mathbb{N}$ such that $\|Y\| / f(s)^{n}<r$. Choosing $T_{1}=s I$, $T_{2}=f(s) s I, T_{3}=f(s)^{2} s I, \ldots, T_{n}=f(s)^{n-1} s I, T_{n+1}=f(s)^{n} X$, we see that

$$
\left(\ldots\left(\left(I \sigma T_{1}\right) \sigma T_{2}\right) \ldots\right) \sigma T_{n+1}=f(s)^{n} f(X) .
$$

The equation $f(s)^{n} f(X)=Y$ clearly has solution $X=f^{-1}\left(Y / f(s)^{n}\right)$. As for the sufficiency, if $A$ is non-invertible then we obtain that all $\left(\ldots\left(\left(A \sigma T_{1}\right) \sigma T_{2}\right) \ldots\right) \sigma T_{n}$ are non-invertible.

In what follows, we compute $A \sigma P$ for an arbitrary positive operator $A \in B(H)^{+}$ and rank-one projection $P$ on $H$. To do so, we recall the notion of the strength of a positive operator along a ray represented by a unit vector. This concept was introduced by Busch and Gudder in [1]. Let $A \in B(H)^{+}$be a positive operator, consider a unit vector $\varphi$ in $H$ and denote by $P_{\varphi}$ the rank-one projection onto the subspace generated by $\varphi$. The quantity

$$
\lambda\left(A, P_{\varphi}\right)=\sup \left\{\lambda \in \mathbb{R}_{+}: \lambda P_{\varphi} \leq A\right\}
$$

is called the strength of $A$ along the ray represented by $\varphi$. According to $[1$, Theorem 4], we have the following formula for the strength:

$$
\lambda\left(A, P_{\varphi}\right)= \begin{cases}\left\|A^{-1 / 2} \varphi\right\|^{-2}, & \text { if } \varphi \in \operatorname{rng}\left(A^{1 / 2}\right) \\ 0, & \text { else. }\end{cases}
$$

(The symbol rng denotes the range of operators and $A^{-1 / 2}$ denotes the inverse of $A^{1 / 2}$ on its range.)

Lemma 2.6. Let $A \in B(H)^{+}$and $P$ be a rank-one projection on $H$. We have $A \sigma P=P \sigma A=f(\lambda(A, P)) P$.

Proof. First assume that $\lambda(A, P)>0$. By (2.1), we have

$$
A \sigma P=\int_{] 0, \infty[} \frac{1+t}{t}\{(t A: P)\} d m(t) .
$$


The parallel sum : is known to be the half of the harmonic mean !. In Lemma 2, [6] we proved that for an arbitrary positive operator $T \in B(H)^{+}$and rank-one projection $P$ on $H$, we have

$$
2(T: P)=T ! P=\frac{2 \lambda(T, P)}{\lambda(T, P)+1} P .
$$

Therefore, denoting $s=\lambda(A, P)$, we can continue (2.5) as follows

$$
\begin{gathered}
A \sigma P=\int_{] 0, \infty[} \frac{1+t}{t} \frac{\lambda(t A, P)}{\lambda(t A, P)+1} P d m(t) \\
=\int_{] 0, \infty[} \frac{1+t}{t} \frac{t \lambda(A, P)}{t \lambda(A, P)+1} P d m(t)=\int_{] 0, \infty[} \frac{1+t}{t} \frac{t s}{t s+1} d m(t) P \\
=s \int_{] 0, \infty[} \frac{(1 / s)(t+1)}{(1 / s)+t} d m(t) P=s f(1 / s) P=f(s) P=f(\lambda(A, P)) P .
\end{gathered}
$$

If $\lambda(A, P)=0$, then in a similar fashion we see $A \sigma P=0=f(\lambda(A, P)) P$.

Lemma 2.7. For any $A, B \in B(H)^{+}$, we have $A \sigma B \neq 0$ if and only if $\operatorname{rng} A^{1 / 2} \cap$ $\operatorname{rng} B^{1 / 2} \neq\{0\}$.

Proof. To see the sufficiency, by the formula (2.4) it follows from $\operatorname{rng} A^{1 / 2} \cap$ $\operatorname{rng} B^{1 / 2} \neq\{0\}$ that there is a rank-one projection $P$ on $H$ and a number $t>0$ such that $t P \leq A, B$. This implies $0 \neq t P=(t P) \sigma(t P) \leq A \sigma B$.

Conversely, assume $A \sigma B \neq 0$. By (2.1), we deduce that $(t A): B \neq 0$ and hence that $(t A) ! B \neq 0$ holds for some $t>0$. It then follows that some positive scalar multiple of a rank-one projection $P$ is less than or equal to $(t A) ! B$. Since $B$ is less than or equal to a scalar multiple of the identity, by the monotonicity property (i) of means this further implies that for some $s>1$, we have $P \leq(s A) !(s I)=s(A ! I)$. Therefore, $2 P /(I+P)=P \leq 2 s A /(I+A)$. The inverse function of $h: t \mapsto 2 s t /(1+t)$, $t \geq 0$ is $k: r \mapsto r /(2 s-r), 0 \leq r<2 s$, which is easily seen to be operator monotone on the interval $[0,2 s[$. It follows that

$$
k(2 P /(I+P)) \leq k(2 s A /(I+A))=A .
$$

One can check that the operator on the left hand side is $k(2 P /(I+P))=(1 /(2 s-1)) P$. This gives us that a positive scalar multiple of $P$ is less than or equal to $A$ implying that the range of $P$ is included in $\operatorname{rng} A^{1 / 2}$. We obtain in a similar fashion that $\operatorname{rng} P \subset \operatorname{rng} B^{1 / 2}$ holds, too. This completes the proof of the lemma.

After these preliminaries, we are now in a position to present the proof of Theorem 2.1.

Proof of Theorem 2.1. By Lemmas 2.4 and 2.5, we see that our transformation $\phi$ preserves the invertible operators in both directions. This means that for any $A \in B(H)^{+}$, we have $A$ is invertible if and only if $\phi(A)$ is invertible. 
It follows that $\phi(I)$ is invertible. By the transfer property, the transformation $\phi(I)^{-1 / 2} \phi(.) \phi(I)^{-1 / 2}$ is a bijective map on $B(H)^{+}$which fulfils $(2.2)$ and sends $I$ to $I$. Therefore, we may and do assume that already our original map $\phi$ satisfies $\phi(I)=I$.

By the characterization of projections (Lemma 2.2) and the order among them (see the sentence before Lemma 2.3), it follows that $\phi$ preserves the projections in both directions as well as the order among them. Consequently, $\phi(0)=0$.

Now, by Lemma 2.7, we see that for any $A, B \in B(H)^{+}$we have $\operatorname{rng} A^{1 / 2} \cap$ $\operatorname{rng} B^{1 / 2} \neq\{0\}$ if and only if $\operatorname{rng} \phi(A)^{1 / 2} \cap \operatorname{rng} \phi(B)^{1 / 2} \neq\{0\}$. One can easily verify that it implies

$$
\operatorname{rng} A^{1 / 2} \subset \operatorname{rng} B^{1 / 2} \Longleftrightarrow \operatorname{rng} \phi(A)^{1 / 2} \subset \operatorname{rng} \phi(B)^{1 / 2} .
$$

From this property, we infer that $\phi$ preserves the (finite) rank of the elements of $B(H)^{+}$.

Let $P$ be a rank-one projection. For every $t>0$, the operator $\phi(t P)$ is of rank one. Since $\phi(t P) \sigma \phi(P)=\phi((t P) \sigma P) \neq 0$, it follows that the range of $\phi(t P)$ has non-trivial intersection with the range of $\phi(P)$. This gives us that $\phi(t P)$ is a scalar multiple of $\phi(P)$. Next we deduce that there exists a bijective function $g_{P}:[0, \infty[\rightarrow[0, \infty[$ such that

$$
\phi(t P)=g_{P}(t) \phi(P)
$$

holds for every $t \geq 0$.

Assume now that $\phi$ is continuous on the scalar multiples of an invertible operator $A \in B(H)^{+}$. Considering the transformation

$$
X \longmapsto \phi(A)^{-1 / 2} \phi\left(A^{1 / 2} X A^{1 / 2}\right) \phi(A)^{-1 / 2},
$$

we obtain a bijective map which satisfies (2.2), sends $I$ to $I$ and is continuous on the set of nonnegative scalar multiples of the identity. Hence, there is no real loss of generality in assuming that this particular operator $A$ equals $I$. In what follows, we do use this assumption.

For any $t \geq 0$, we have

$$
\phi(t I) \sigma \phi(P)=\phi((t I) \sigma P)=\phi(f(t) P)=g_{P}(f(t)) \phi(P) .
$$

By the formula (2.3), for an arbitrary $B \in B(H)^{+}$, the transformation $C \mapsto C \sigma B$ is norm continuous on the set of all invertible elements of $B(H)^{+}$. We deduce that $g_{P}(f(t)) \rightarrow g_{P}\left(f\left(t_{0}\right)\right)$ whenever $t_{0}>0$ and $t \rightarrow t_{0}$. Applying (iii), the same follows for $t_{0}=0$, too. Therefore, we obtain that $g_{p}$ is continuous on the range of $f$. Since 
$g_{P}$ is a bijection of the nonnegative reals and $g_{P}(0)=0, g_{P}(1)=1$ obviously hold, it follows that $g_{P}$ is strictly increasing on the range of $f$.

We show that $\phi$ preserves the order of positive operators in both directions. We compute on one hand

$$
\phi(A \sigma P)=\phi(f(\lambda(A, P)) P)=g_{P}(f(\lambda(A, P))) \phi(P),
$$

while on the other hand we have

$$
\phi(A \sigma P)=\phi(A) \sigma \phi(P)=\lambda(\phi(A), \phi(P)) \phi(P) .
$$

We obtain that $g_{P}(f(\lambda(A, P)))=\lambda(\phi(A), \phi(P))$ holds for any $A \in B(H)^{+}$and rankone projection $P$. Now, for given $A, B \in B(H)^{+}$and arbitrary rank-one projection $P$ on $H$, we have

$$
\begin{gathered}
\lambda(A, P) \leq \lambda(B, P) \Leftrightarrow g_{P}(f(\lambda(A, P))) \leq g_{P}(f(\lambda(B, P))) \\
\Leftrightarrow \lambda(\phi(A), \phi(P)) \leq \lambda(\phi(B), \phi(P)) .
\end{gathered}
$$

We learn from [1] that $A \leq B$ holds if and only if $\lambda(A, P) \leq \lambda(B, P)$ holds for every rank-one projection $P$ on $H$. Therefore, it follows that we have $A \leq B$ if and only if $\phi(A) \leq \phi(B)$. This means that $\phi$ is an order automorphism of $B(H)^{+}$. The structure of such transformations was described in [3]. We proved there that every such map is implemented by an invertible bounded linear or conjugate-linear operator. Consequently, it follows that $\phi$ is of the form

$$
\phi(A)=T A T^{*}, \quad A \in B(H)^{+}
$$

with an invertible bounded linear or conjugate-linear operator $T$ on $H$, and this completes the proof of the theorem in the present case.

Suppose now that $\phi$ maps the scalar multiples of an invertible operator $A$ into scalar multiples of $\phi(A)$. Considering the transformation

$$
T \longmapsto \phi(A)^{-1 / 2} \phi\left(A^{1 / 2} T A^{1 / 2}\right) \phi(A)^{-1 / 2}
$$

just as above, we see that there is no loss of generality in assuming that this particular operator $A$ equals the identity. In what follows, we assume that it is really the case. It means that there is an injective function $g:[0, \infty[\rightarrow[0, \infty[$ such that $\phi(t I)=g(t) I$, $t \geq 0$.

We compute

$$
\phi(f(t) P)=\phi((t I) \sigma P)=(g(t) I) \sigma \phi(P)=f(g(t)) \phi(P) .
$$


Now, for an arbitrary $B \in B(H)^{+}$, we compute $B \sigma P=f(\lambda(B, P)) P$ implying $\phi(B \sigma P)=\phi(f(\lambda(B, P)) P)=f(g(\lambda(B, P))) \phi(P)$. On the other hand, we have

$$
\phi(B \sigma P)=\phi(B) \sigma \phi(P)=f(\lambda(\phi(B), \phi(P))) .
$$

Therefore, by the injectivity of $f$, it follows that

$$
g(\lambda(B, P))=\lambda(\phi(B), \phi(P))
$$

holds for all $B \in B(H)^{+}$and rank-one projection $P$ on $H$. Pick an invertible $B \in$ $B(H)^{+}$and consider the set of all $\lambda(B, P)$ where $P$ runs through the connected set of all rank-one projections. By the formula (2.4), it is easy to see that this set is an interval. Taking into account the equality (2.6), it follows that the injective function $g:[0, \infty[\rightarrow[0, \infty[$ maps every interval $[\alpha, \beta]$ with $0<\alpha<\beta<\infty$ onto an interval. It apparently yields that the restriction of $g$ onto the open interval $] 0, \infty[$ is strictly monotone. Assume for a moment that this function is strictly monotone decreasing. Let $A, B \in B(H)^{+}$be invertible and $P$ be an arbitrary rank-one projection on $H$. Then we infer

$$
\begin{gathered}
\lambda(A, P) \leq \lambda(B, P) \Leftrightarrow g(\lambda(B, P)) \leq g(\lambda(A, P)) \\
\Leftrightarrow \lambda(\phi(B), \phi(P)) \leq \lambda(\phi(A), \phi(P)) .
\end{gathered}
$$

This means that $\phi$, when restricted onto the set of invertible elements of $B(H)^{+}$, is an order reversing automorphism. Considering the transformation $A \mapsto \phi(A)^{-1}$ we obviously obtain an order automorphism of that set. By a result in [7], the structure of those transformations is just the same as that of the order automorphisms of the whole set $B(H)^{+}$. That is, they are implemented by invertible bounded linear or conjugate-linear operators on $H$. Since here we also have that the identity is sent to the identity, it follows easily that $\phi$ is of the form $\phi(A)=U A^{-1} U^{*}$ with a unitary or antiunitary operator $U$ on $H$. It implies that the inverse operation satisfies $(2.2)$, i.e., we have $(A \sigma B)^{-1}=A^{-1} \sigma B^{-1}$ for all invertible $A, B \in B(H)^{+}$. Putting $A=I$ and $B=s I$, this immediately gives us that $1 / f(s)=f(1 / s), s>0$. But the mean $\sigma$ is symmetric implying that $s f(1 / s)=f(s)$ holds for all $s>0$. It follows trivially that we necessarily have $f(s)=\sqrt{s}, s>0$, i.e., $\sigma$ is the geometric mean. But the bijective maps on $B(H)^{+}$preserving the geometric mean have been described in [5]. The result presented there shows that those maps just coincide with the order automorphisms of $B(H)^{+}$. But this contradicts the fact above that $\phi$ is an order reversing automorphism of the set of all invertible elements of $B(H)^{+}$. It yields that the case where the restriction of $g$ onto the open interval $] 0, \infty[$ is strictly monotone decreasing is untenable. It remains that $g$ is strictly monotone increasing on $] 0, \infty[$ and hence also on $[0, \infty[$. Using (2.6), we can see just as before that $\phi$ is an order automorphism of $B(H)^{+}$and then complete the proof as in the first case. 
We conclude the paper with a result describing the bijective transformations of $B(H)^{+}$which preserve the norm of means of operators. The theorem below shows that every such map originates from an isometric linear or conjugate-linear *-algebra automorphism of $B(H)$.

ThEOREM 2.8. Let $\phi: B(H)^{+} \rightarrow B(H)^{+}$be a bijective map with the property that

$$
\|\phi(A) \sigma \phi(B)\|=\|A \sigma B\|
$$

holds for every $A, B \in B(H)^{+}$. Then there exists either a unitary or an antiunitary operator $U$ on $H$ such that $\phi$ is of the form

$$
\phi(A)=U A U^{*}, \quad A \in B(H)^{+} .
$$

Proof. First observe that $\phi(0)=0$. Indeed, it follows from

$$
\|\phi(A)\|=\|\phi(A) \sigma \phi(A)\|=\|A \sigma A\|=\|A\|
$$

meaning that $\phi$ is norm-preserving.

The assumptions in the theorem imply that for any $A, B \in B(H)^{+}$, we have $A \sigma B \neq 0$ if and only if $\phi(A) \sigma \phi(B) \neq 0$. Just as in the proof of Theorem 2.1, we infer that $\operatorname{rng} A^{1 / 2} \cap \operatorname{rng} B^{1 / 2} \neq\{0\}$ holds if and only if $\operatorname{rng} \phi(A)^{1 / 2} \cap \operatorname{rng} \phi(B)^{1 / 2} \neq\{0\}$ which then implies that

$$
\operatorname{rng} A^{1 / 2} \subset \operatorname{rng} B^{1 / 2} \Longleftrightarrow \operatorname{rng} \phi(A)^{1 / 2} \subset \operatorname{rng} \phi(B)^{1 / 2} .
$$

We can proceed showing that $\phi$ necessarily preserves the rank-one operators in both directions. As $\phi$ preserves the norm, too, it follows that $\phi$ preserves the rank-one projections in both directions. For any rank-one projection $P$ on $H$, we have

$$
f(\lambda(\phi(A), \phi(P)))=\|\phi(A) \sigma \phi(P)\|=\|A \sigma P\|=f(\lambda(A, P))
$$

implying $\lambda(\phi(A), \phi(P))=\lambda(A, P)$. Just as in the proof of Theorem 2.1, this gives us that $\phi$ is an order automorphism of $B(H)^{+}$and hence it is of the form

$$
\phi(A)=T A T^{*}, \quad A \in B(H)^{+}
$$

with an invertible bounded linear or conjugate-linear operator $T$ on $H$. Referring again to the property that $\phi$ preserves the norm, one can easily deduce that $T$ is in fact either a unitary or an antiunitary operator. This completes the proof of the theorem. $\square$ 


\section{REFERENCES}

[1] P. Busch and S.P. Gudder. Effects as functions on projective Hilbert spaces. Lett. Math. Phys., 47:329-337, 1999

[2] F. Kubo and T. Ando. Means of positive linear operators. Math. Ann., 246:205-224, 1980.

[3] L. Molnár. Order-automorphisms of the set of bounded observables. J. Math. Phys., 42:59045909, 2001.

[4] L. Molnár. Selected Preserver Problems on Algebraic Structures of Linear Operators and on Function Spaces. Lecture Notes in Mathematics, Vol. 1895, Springer-Verlag, Berlin, 2007.

[5] L. Molnár. Maps preserving the geometric mean of positive operators. Proc. Amer. Math. Soc., 137:1763-1770, 2009

[6] L. Molnár. Maps preserving the harmonic mean or the parallel sum of positive operators. Linear Algebra Appl., 430:3058-3065, 2009.

[7] L. Molnár. Order automorphisms on positive definite operators and a few applications. Linear Algebra Appl., 434:2158-2169, 2011.

[8] D. Petz. Monotone metrics on matrix spaces. Linear Algebra Appl., 244:81-96, 1996. 